\title{
Development of farm pond operational modeling using Neuro-Fuzzy technique
}

\author{
Ashish V. Sonawane ${ }^{1 *}$, Murtaza Hasan ${ }^{2}$ and Deepak Singh ${ }^{1}$ \\ ${ }^{1}$ College of Agricultural Engineering and Technology, Dediapada, Navsari Agricultural University, Navsari \\ -393040 (Gujarat), INDIA \\ ${ }^{2}$ Center for Protected Cultivation Technology, Indian Agricultural Research Institute, New Delhi-110012, INDIA \\ *Corresponding author. E-mail: ashishswe@nau.in
}

Received: September 24, 2015; Revised received: February 9, 2016; Accepted: May 7, 2016

\begin{abstract}
Study was conducted to derive operational model for a farm pond of 3000 cubic meter capacity at Center for protected cultivation technology (CPCT), Indian Agricultural Research Institute, New Delhi, India which was the important source of irrigation water of the farm of the area 10 ha. The Neuro-Fuzzy approach was used to develop the operational model and to derive operational rules for proper irrigation scheduling of the horticultural crops grown at CPCT. Based upon the inputs like crop water requirement, evaporation losses and farm pond inflow the model predicting outflow of the reservoir was developed. The developed model was having high accuracy and predictability when tested statistically. The coefficient of determination $\left(R^{2}\right)$ was found to be 0.96 , whereas the model efficiency (E) was 0.97 which shows the high reliability of the model. The operating rules which were of 'If-Then' form were also developed which would lead to better management of the farm pond system and would also improve the irrigation scheduling at CPCT farm, IARI, New Delhi.
\end{abstract}

Keywords: Farm pond, Irrigation scheduling, Neuro-Fuzzy, Operational rules

\section{INTRODUCTION}

Farm pond or on-farm reservoir (OFR) is the most important component of water resource network at the village or farm level. It is used for the storage of rainfall generated surface runoff and is located at the downstream side of the watershed. The two main important uses of farm pond are storage of precious rainfall generated surface runoff water and ground water recharge (Sonawane et al., 2014). The storage water is subsequently used for irrigation of different crops located around the farm pond. Farm pond plays a very important role in the maintenance of optimum level of ground water table and quality management of ground water at the micro level or village level. Government of India has recently started many schemes through MNREGA (Mahatma Gandhi National Rural Employment Guarantee Act) to revive the old ponds and construct useful asset like farm ponds at the village level. NREGA and ICAR (Indian Council of Agricultural Research) has established convergence guidelines for improving the quality of works planning and execution listed under NREGA. Construction of farm ponds for water conservation has been listed as a major activity under NRM (Natural Resource Management) related guidelines (Anonymous, 2008).

Unlike farm ponds, reservoirs having large catchment and command area, play an important role for the water resource management at the macro level (Chaves et al., 2007). The multipurpose reservoir has been in used since ages for the irrigation, power generation and other uses. Their operational management strategies have been standardized and already implemented at the ground level (Sonawane et al., 2014). For modeling of complex water resource system network problems, the soft computing techniques (like fuzzy logic, ANN, GA, etc) have been employed extensively as these technique handle uncertainty and ambiguity in effective manner by imitating human way of reasoning and decision making (Sonawane et al., 2013). To handle such problems when conditions of the systems are uncertain, these techniques have proved their effectiveness (Mehta and Jain, 2009). Therefore soft computing techniques can be are very well adapted to develop farm pond management strategy as farm ponds are more susceptible to poor distribution of water due to highly uncertain demand, supply and storage. In the present study, efforts have been made to employ Neuro -Fuzzy technique to determine the operating rules of the farm pond.

However, there seems to have no study which is undertaken to develop management strategies for OFR. The soft computing modeling of farm pond at the micro level is urgently required for judiciously and efficiently utilizing the precious water and energy required for pumping. It will lead to efficient management of farm pond and optimum irrigation scheduling of different crops. Consequently the study was carried with the main objective that to develop 
farm pond operational model using Neuro-Fuzzy technique (Sonawane, 2011). for effective irrigation scheduling of horticultural crops at Center for protected cultivation Technology (CPCT) farm of IARI, New Delhi

\section{MATERIALS AND METHODS}

Study area: The experiment was carried out at center for protected cultivation technology (CPCT), Indian Agricultural Research Institute, (IARI), New Delhi located between latitudes of $28^{\circ} 37^{\prime} 22^{\prime}, \mathrm{N}$ and $28^{\circ}$ $38^{\prime} 39^{\prime}$ ' $\mathrm{N}$ and longitudes of $77^{\circ} 08^{\prime} 45^{\prime}, \mathrm{E}$ and $77^{\circ}$ $10^{\prime} 24^{\prime \prime}$ E at an average elevation of 230 meter above mean sea level (MSL). The 10 hectare farm consisted of different green houses, shed nets, nurseries, open fields, orchard and $3000 \mathrm{~m}^{3}$ capacity farm pond. The pond is fed by harvested runoff during the rainfall events and by pumping the ground water and also. This farm pond is the only source of irrigation for entire CPCT farm. Pond is trapezoidal shaped dug-out type pond and is lined by concrete to avoid seepage losses through sides and bottom of the pond.

Soft computing techniques: Concept of soft computing was introduced by Lotfi Zadeh in 1965. The soft computing is inspired by human neural system and is excellent in achieving tractability and has robustness with low solution cost. It can handle the uncertainty and partial truth effectively. Some of the important techniques are fuzzy logic, Artificial neural network, Genetic algorithm, etc.

Fuzzy Logic (FL): Fuzzy logic is generally considered to be a logical system. FL generalizes classical two-valued logic for reasoning under uncertainty (Yen and Langari 2003). FL works on fuzzy set theory due to which it is a powerful tool for handling handles uncertainties and partial truth effectively by mathematical methods. Fuzzy set theory deals with using ambiguous meaning of the natural linguistic terms in valuation and reasoning (Hong and Lee, 1996). It was first used in system control application by Mamdani in 1974.

Fuzzy set is defend as, if ' $\mathrm{S}$ ' is a collection of objects denoted generally by ' $S$ ' then a fuzzy set $A$ in $\mathrm{S}$ is a set of ordered pairs as shown in Eq. 1:

$A=\left\{\left(s, \mu_{\mathrm{A}}(s)\right) \mid s \in \mathrm{S}\right\}$

Where $\mu_{\mathrm{A}}(s)$ is a membership function (degree of truth) of ' $S$ ' in $A$. It also indicates the interval or membership space of real numbers from 1 to 0 . If the membership space contains two points 0 and 1 only, $A$ is non fuzzy (crisp) and $\mu_{\mathrm{A}}(s)$ is identical to characteristics function of crisp set (Zimmermann, 1996). FL system describes process in terms of linguistic variables and If-Then rules. Each linguistic variable is represented by different terms $\left({ }_{i}{ }_{i}\right)$ where $i$ indicates the linguistic variable, $\mathrm{i}=1,2 . \ldots ., \mathrm{n}$; and $\mathrm{j}$ indicates the term of $i$-th linguistic variable, $j=1,2, \ldots . ., m_{i}$, and $\mathrm{m}_{\mathrm{i}}$ is the number of terms of $\mathrm{i}^{\text {th }}$ linguistic variable. Each term ${ }_{i}{ }_{i}$ is described by its membership function $\left(\mu_{i}{ }_{i}\right)$ and each crisp value of linguistic variable will have a degree of membership ${ }_{i}{ }^{j}$.

Process of decision making in FL involves three stages viz.,

Fuzzification

Fuzzy inference

Defuzzification

In the fuzzification process, the entire crisp input values translated into linguistic terms. Membership functions of various shapes like triangular, trapezoidal, Gaussian shapes described by each of the linguistic terms. The degree of membership is represented by ' $\mathrm{V}$ '

Computation of fuzzy rule consequences is performed in fuzzy interface. In fuzzy system the relationship between input and output in linguistic terms are represented using fuzzy rules. The rules consist of precondition (If-part) and consequences (Then-part). Defuzzification is the process where the fuzzy output is converted in to crisp values using membership functions. Center of Area (CoA) and Center of Maximum (CoM) is the widely used membership function.

In the present study Center of maximum is selected for defuzzification. It computes the crisp output as a weighted average of the center of membership function $\left(c_{i}{ }^{j}\right)$, weighted by the inference results $\left(\mu\right.$ RESULT $\left.{ }_{i}\right)$ as shown in Eq 2.

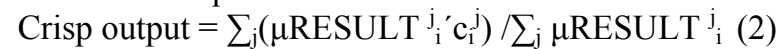

Artificial neural network (ANN): ANN is popularly known as replicated model of human brain system. It functions as parallel distributed network which is composed of neurons, which imitate the brain nerve cells. ANN should first train using training algorithms. It has excellent capability to learn new associations, new patterns and new dependencies. ANN represents the new generation of information processing networks (Fuller, 1999).

The basic structure of ANN is shown in Fig. 2, it consists of three layers viz. input, hidden and output layer. Each neuron processes each incoming inputs into an output. The output is then again connected to other neurons which form net of neurons. The information enters the net at the input layer. All layers of the net, process these signals through the net until and unless they reach to the output layer.

Assume the inputs of certain neuron are $\left(\mathrm{X}_{1}, \mathrm{X}_{2} \ldots \ldots\right.$.... $\left.X_{n}, w_{1}, w_{2} \ldots \ldots W_{n}\right)$ where $X_{i}$ denotes an $i^{\text {th }}$ input, $w_{i}$, represents the $i^{\text {th }}$ connection weight, and $n$ represents the number of the neurons input connections. Each node produces an output value $\mathrm{O}$.

The process of transformation of input value is described by two functions as follows:

Int $=\mathrm{S} \mathrm{w}_{\mathrm{i}} \mathrm{X}_{\mathrm{i}},(\mathrm{i}=1$ to $\mathrm{n})$

Act $=1 /\left(1+\mathrm{e}^{- \text {-nt }}\right)$

Where, Int is the standard form of the propagation function that performs a weighted sum for inputs and Act is standard form of activation function that computes the neuron's output. 


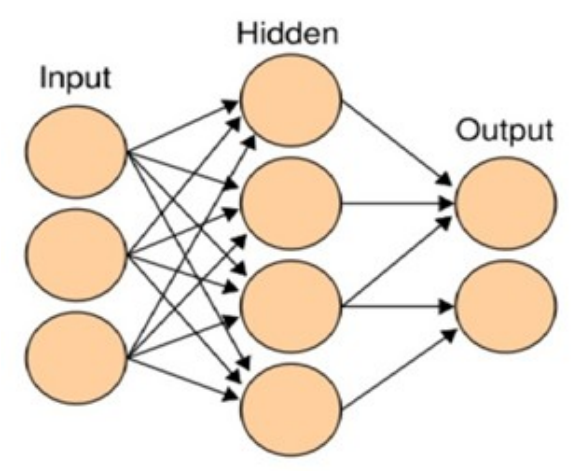

Fig. 1. Generalized structure of neural network.

Neuro-Fuzzy system: Neuro-Fuzzy System is integration of FL and ANN, which combines advantages of both in one system and simultaneously devoid of the weakness (Hasan, 2007). ANN is called as 'Black box' model as, it is good in acquiring knowledge automatically but fails to explain how it performs and reaches outputs. On the other hand FL is effective tool in handling imprecise and uncertain situations in as understandable and transparent logical structure (IfThen), but it is not good in acquiring the knowledge of the relationship between the data. Integration approach of ANN and FL was classified into three groups by Kruse and Nauck (1995). It is very popular because it can learn governing relations from given data and the fuzzy rules obtained can provide the linguistic description for the working of the model (Tutmez et al., 2006). The three groups are: Concurrent Neuro-Fuzzy Model, Cooperative Neuro-Fuzzy Model and Hybrid NeuroFuzzy Models. Hybrid Neuro-Fuzzy Models is the strongest form and Adaptive Neuro-Fuzzy Inference System (ANFIS) comes under this category.

In general sense the the input layer of the model deals with the fuzzification process, the hidden layer functions as fuzzy rules base whereas the output layer performs defuzzification.

Three important steps in development of Neuro-Fuzzy model consist of training, testing and validation. According to availability of the data, it should be divided into three parts to complete modeling procedure. For the sake of pattern recognition between input and output, various combinations of data are provided into training step to Nero-Fuzzy network. Various modifications in internal representation are carried out in testing phase. In validation the statistical tests for developed model is carried out for its predictability and acceptability.

Adaptive Neuro-Fuzzy inference system (ANFIS): ANFIS was used for system identification to find appropriate network architecture and a set of parameters, which can best model an unknown target system containing input-output data sets (Jang, 2005). Jang and Sun (1995) had developed an adaptive network based fuzzy inference system which is commonly known as ANFIS. It is very popular because it can learn governing relations from given data and the fuzzy rules obtained can provide the linguistic description for the working of the model (Tutmez et al., 2006) Data Preparation and standardization: Sensitivity analysis was carried out to select only relevant parameters and to remove non relevant parameters. The parameters which affect the output are only selected for model development. The parameters which remains constant throughout or which did not change significantly should not be taken into consideration for model development. Daily data for crop water requirement, evaporation losses, inflow and outflow of farm pond were collected for the years 2001 to 2010 . Out of the data used for model development $60 \%$ of the data were used for training, $20 \%$ for testing and remaining $20 \%$ were used for validation of the model.

Development of Neuro-Fuzzy model: For developing Neuro-Fuzzy model the computer programme was written in MATLAB 7.5 compatible language for predicting outflow. Sugeno type Fuzzy Inference System (FIS) was used which improves the efficiency of defuzzification process. All relevant inputs (cropwater requirement, pan evaporation, farm pond inflow) and output (outflow) were used to develop the model. Model was trained with a set of known input and output data. The training was repeated with sets of shuffled data. The RMSE was noted down for each analysis and cross validation was done to determine the $\mathrm{R}^{2}$ values. When an optimum prediction statistic was obtained in relation to epoch size and cross-validation results, the learning process was terminated. Three dimensional views of input and output were developed.

Model evaluation: For evaluation of model the model efficiency factor (E) and coefficient of determination $\left(\mathrm{R}^{2}\right)$ were determined. Model efficiency and coefficient of determination are important parameters which determine the applicability of the developed model.

\section{RESULTS AND DISCUSSION}

Development of Neuro-Fuzzy model: Neuro-Fuzzy model was developed by using three input parameters namely, crop water requirement, pan evaporation and farm pond inflow. Development of Neuro-Fuzzy model included integration of fuzzy logic (FL) and Artificial neural network (ANN) and development of ANFIS.

Development of ANFIS: For the study purpose Sugeno type FIS was selected because it produces crisp output. ANFIS consists of Sugeno type FIS, membership functions of input parameters and fuzzy rules. Gaussian membership function was used for defining fuzzy sets of input parameters by normalizing input parameters into membership between 0 and 1 . Fuzzy rules were determined for input parameters using membership function and known value of output. Hasan,(2007) followed the similar methodology to developed ANFIS system for irrigation scheduling. 
Sugeno type FIS: Sugeno type of FIS was selected as it was found that, efficiency of de-fuzzification was higher in Sugeno type of FIS as compared to Mamdani type FIS. This kind of superiority of Sugeno FIS was also reported by Jang and Sun (1995) and Hasan (2007). The Sugeno type FIS which was developed is shown in fig. 1 it was developed for farm pond outflow on the basis of input parameters. MATLAB 7.5 was used for this purpose. Fig. shows that there were there were three layers viz., input, intermediate layer (Inference system) and output layer. Intermediate layer performs the fuzzification of crisp inputs and defuzzification of linguistic output. The three inputs were crop water requirement, pan evaporation and inflow of farm pond from which the outflow of farm pond was predicted as output.

Generation of membership functions: For the Sugeno type FIS the different membership functions were generated. The Gaussian membership function (gaussmf) was developed for input parameters like water requirement (WR), Evaporation and Inflow of the farm pond and for which the system output as outflow of pond as shown in Fig. 2. The FIS was developed by training and testing of long term data. The FIS rules were generated by using ANFIS. Three membership functions viz., low, medium and high (in $1 \mathrm{mf} 1$, in $1 \mathrm{mf} 2$, in $1 \mathrm{mf} 3$ etc.) with membership ranging from 0 to 1 were selected based on close association between observed and predicted values. The membership functions are shown in Figs. 2 to 4 .

Generation and selection of fuzzy rules: As reported by Chaves and Kojiri (2007) fuzzy rules are the unique and very important components of ANFIS. ANFIS used the logic of human interpretaion of the proceseess. The fuzzy sets used in fuzzy rules are represented by membership functiuon which is chosen to indiacate the qulaities of consequent. Output of each rule is indiacted by a fuzzy set. Output fuzzy sets for each rulle was agregated into ingle fuzzy set. The output set is then defuzzified and/or resolved into single one.

Corresponding to the membership function 27 rules were derived out of which 4 best rules were selected manually. The developed rules were in the form of 'IfThen' and these rules transformed the fuzzy inputs to fuzzy outputs. Fig. 5 and 6 shows the fuzzy rules developed by ANFIS. Linguistic rules given by ANFIS are very easy to understand and helps in effective operation of farm pond.

Fuzzy rules for operating policy of farm pond

- If (WR is low) and (Evaporation is high) and (Inflow is low) then outflow is low

- If (WR is high) and (Evaporation is low) and ( Inflow is high) then outflow is high

- If (WR is medium) and (Evaporation is high) and

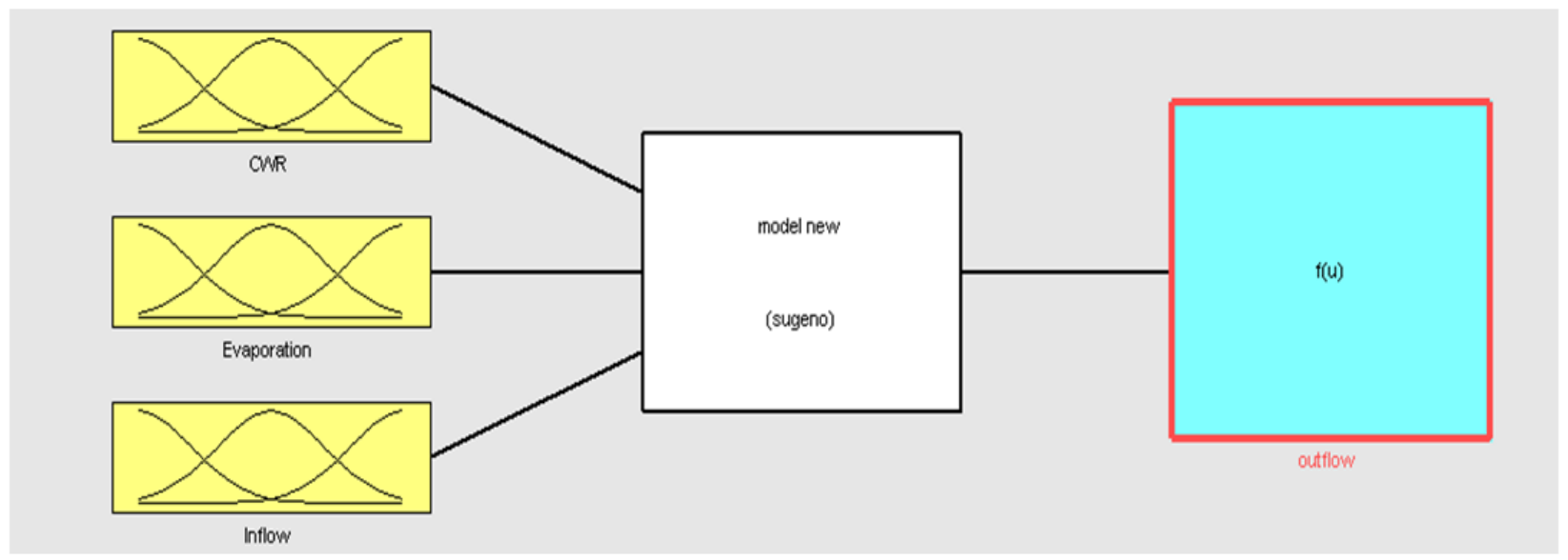

Fig. 1. Sugeno type FIS.

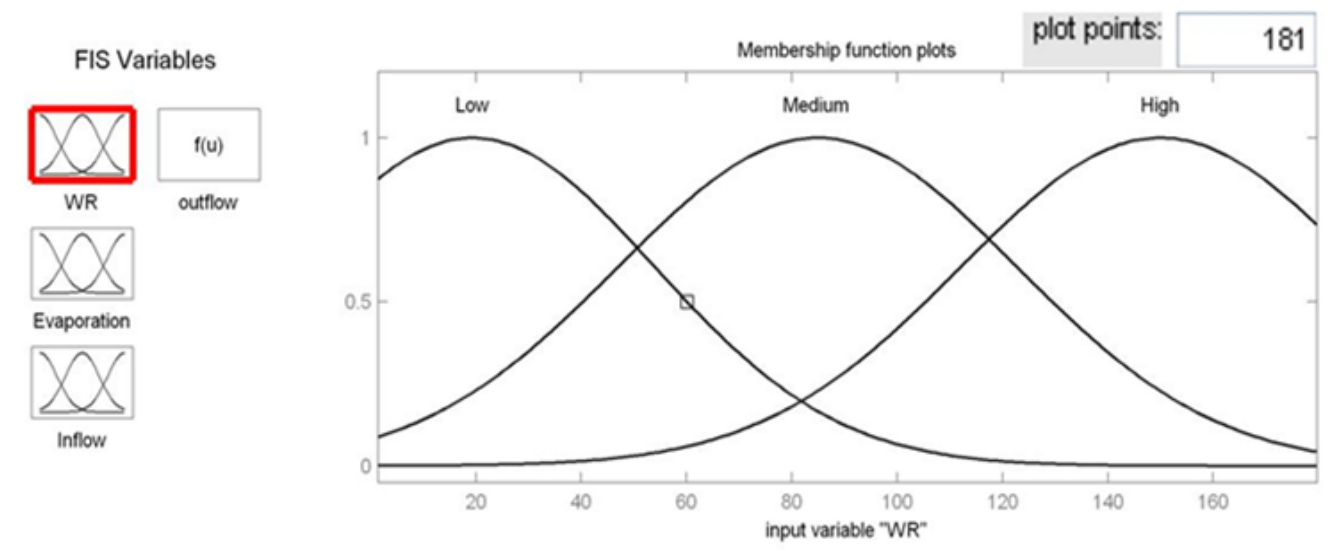

Fig. 2. Membership function for input variable water requirment (WR). 

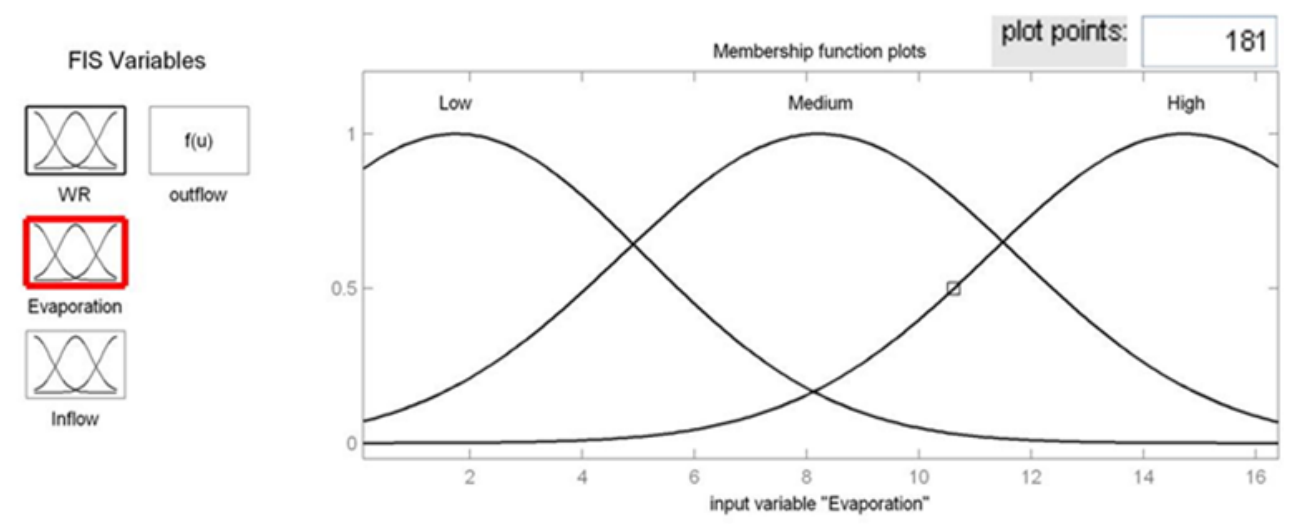

Fig. 3. Membership function for input variable evaporation.

FIS Variables

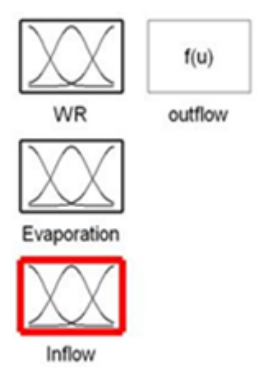

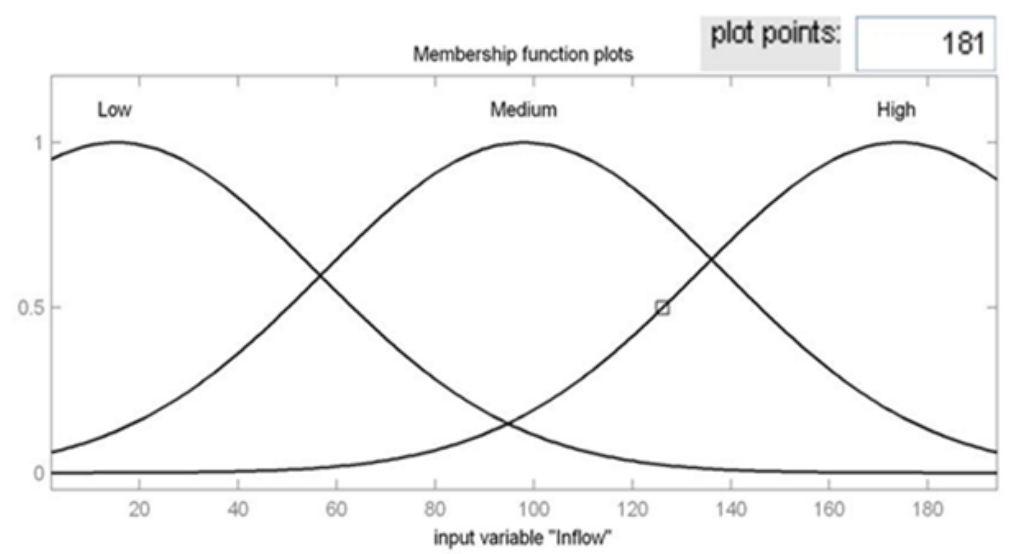

Fig. 4. Membership function for input variable inflow.

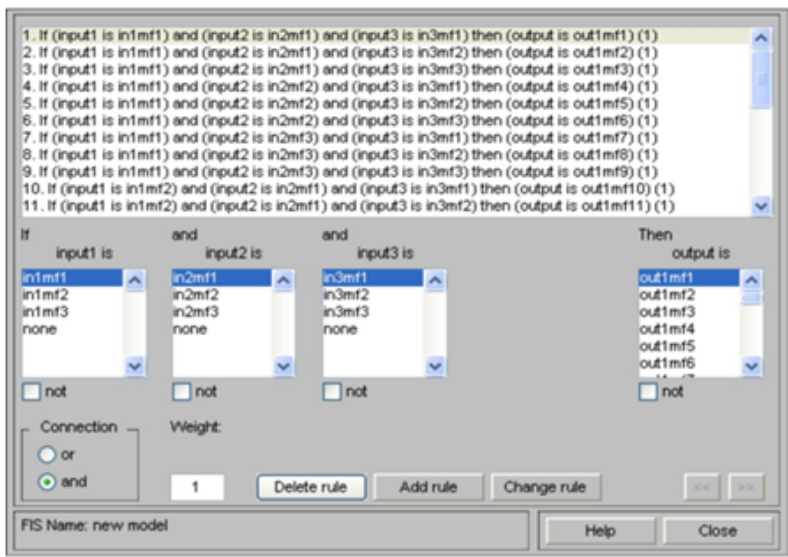

Fig. 5. Fuzzy rule editor for Neuro-Fuzzy model.

( Inflow is low) then outflow is low

- If (WR is medium) and (Evaporation is high) and ( Inflow is medium) then outflow is medium

The final structure of Neuro-Fuzzy farm pond operational model is shown in Fig. 7.

Validation of model: The farm pond operational model developed by using Neuro-Fuzzy technique was validated using new sets of input and output data. Predicted values were compared with the observed/ actual value. The result of validation is shown in Fig. 8 , which revealed that the developed Neuro-Fuzzy

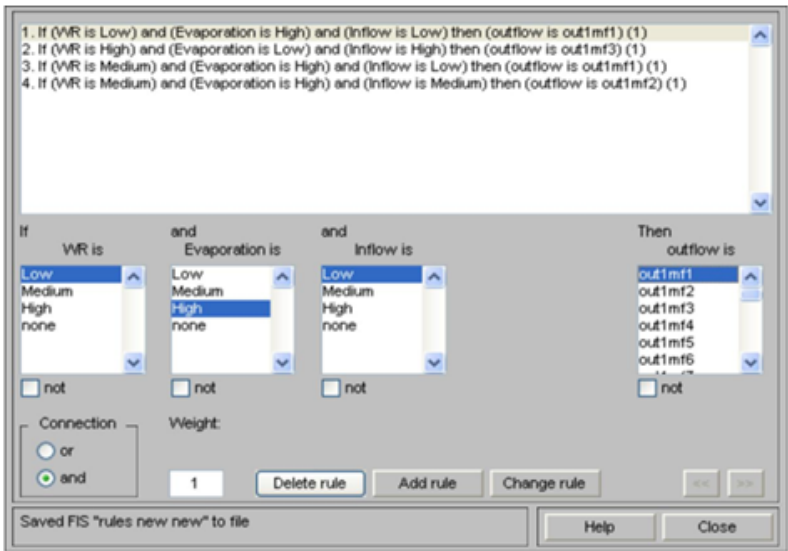

Fig. 6. Fuzzy rule for operating policies of farm pond.

model was able to predict outflow with high accuracy. Coefficient of determination $\left(\mathrm{R}^{2}\right)$ between observed and predicted value was found to be 0.96 . The model efficiency (E) was also found to be very high i.e. 0.97 which showed the highly efficient model

In this way the operational model of the farm pond was developed using the Neuro-Fuzzy approach and was observed to be efficient statistically. The operating rules of farm pond were also developed which would lead to proper management of the farm pond system at CPCT farm by avoiding misdistribution of water. 


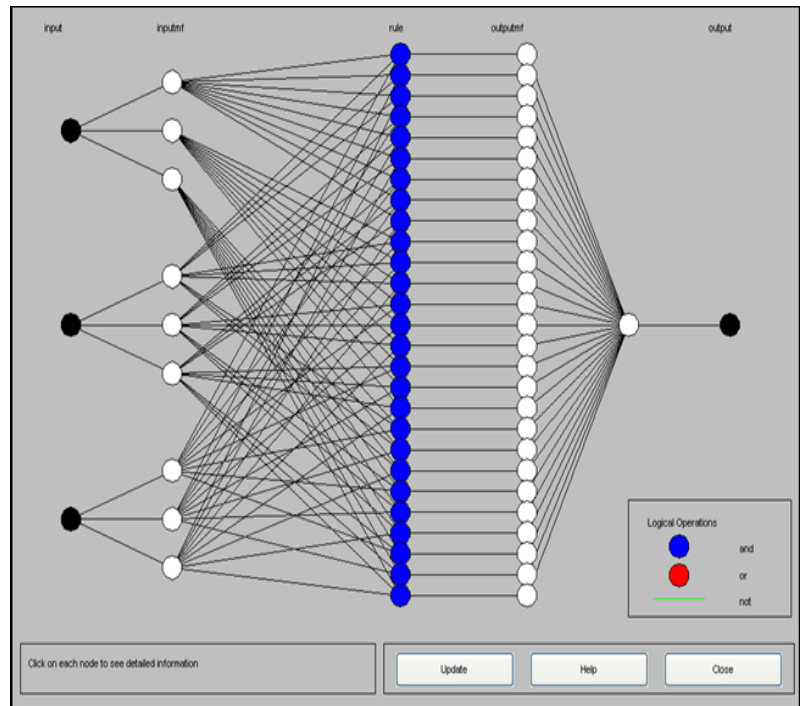

Fig. 7. Neuro-Fuzzy farm pond operational model.

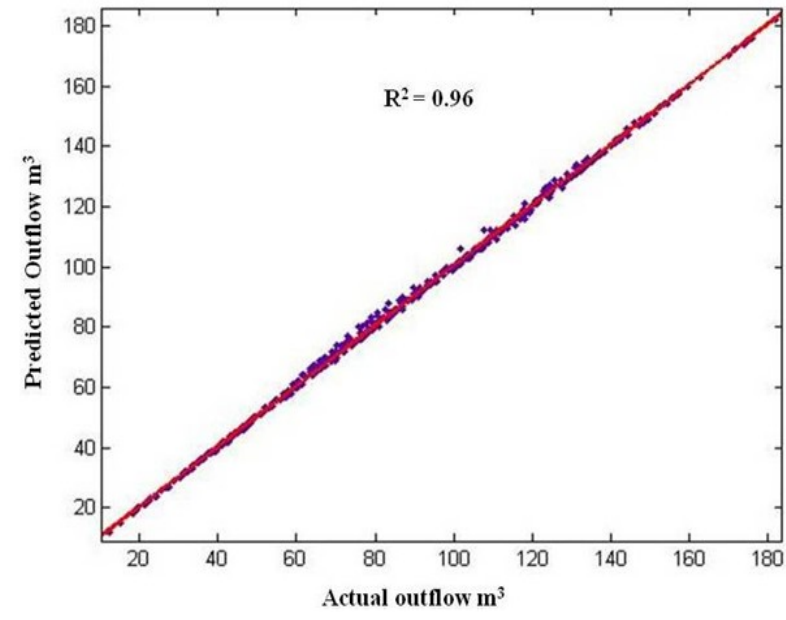

Fig. 8. Validation of model for prediction of farm pond outflow.

\section{Conclusion}

Operating polices of the farm pond at CPCT farm, CPCT, IARI, New Delhi was determined. Crop water requirement, evaporation loss and inflow were used to determine optimum outflow of the farm pond. NeuroFuzzy approach was used to determine the operating policies of the farm pond in linguistic form which was in 'if-then' format. The developed model was validated statistically which showed that the model has very high predictability as the coefficient of determination $\left(\mathrm{R}^{2}\right)$ between observed and predicted value was found to be 0.96 . The model efficiency was also found to be high (0.97). The operating policies developed for the farm pond operation may lead to sustainable water management at field level.

\section{ACKNOWLEDGEMENT}

The authors wish to acknowledge the support and guidance given by the faculty of Division of Agricultural Engineering and Center for Protected Cultivation Technology, (CPCT), Indian Agricultural Research Institute, New Delhi, India and College of Agricultural Engineering and Technology, Navsari Agricultural University, Navsari Gujarat.

\section{REFERENCES}

Anonymous (2008). Guidelines on convergence with national rural employment scheme. Department of rural development, Ministry of Rural Development, Govt. of India, pp 19-34.

Chaves, P. and Kojiri, T. (2007). Stochastic fuzzy neural network: case study of optimal reservoir operation. Journal of Water Resources Planning and Management 133(6):509-518.

Fuller, R. (1999). Introduction to Neuro-Fuzzy Systems. Physica-Verlag, Heidelberg, pp 283.

Hasan, M. (2007). Fuzzy-Neuro model for drip irrigation scheduling of greenhouse rose. Ph.D. thesis, Indian Agricultural Research Institute, New Delhi, India.

Hong, T.P. and Lee, C.Y. (1996). Introduction of fuzzy rules and membership functions from training examples. Fuzzy sets and Systems 84:33-47.

Jang, J.S.R. and Sun, C.T. (1995). Neuro-fuzzy modeling and control. Proc. IEEE 83(3): 378-406.

Jang, J.S.R. (2005). Adaptive network in Neuro-Fuzzy and soft computing. Pearson Education, New Delhi. pp: 225 -251 .

Kruse, R. and Nauk, D. (1995). Learning methods of for fuzzy system. Proc. of the 3rd German-GI workshop Neuro-Fuzzy Systems, Darmstadt, Germany, Nov. 1517.

Mehta, R. and Jain, S.K. 2009. Optimal operation of a multipurpose reservoir using Neuro-fuzzy technique. Water resource management 23: 509-529.

Sonawane, A.V. (2011). Development of farm pond operational model for irrigation scheduling of horticultural crops. Unpublished thesis, Indian Agricultural Research Institute, New Delhi. India.

Sonawane A., Desai, S., Rajurkar, G. and Singh, D. (2014). Soft computing approach for optimal reservoir operation. Journal of Soil and Water Conservation, 13(1):83-88.

Sonawane A., Hasan, M., Rajwade, Y., Desai, S., Rajurkar, G., Shinde, V., Singh, D. and Singh, M. (2013). Comparison of Neuro-Fuzzy and regression models for prediction of outflow of an on-farm reservoir. International Journal of Agriculture, Environment \& Biotechnology, 6(2): 187-193.

Tutmez, B., Hatipogolu, Z. and Kaymak, U. (2006). Modelling of electrical conductivity of ground water using and adaptive neuro-fuzzy inference system. Computers and Geosciences. 32:421-433.

Yen, J. and Langari, R. (2003). Fuzzy logic: Intelligence, Control and Information. Pearson Education, New Delhi.

Zimmermann, H.J. (1996). Fuzzy set theory and its application. Kluwer Academic Publisher, Boston, London. 